\title{
The Development of a Mindful-Eating Intervention Program Among Third Through Fifth Grade Elementary School Children and Their Parents
}

\author{
Shannon Pierson ${ }^{1}$, Keiko Goto ${ }^{1}$, Joan Giampaoli ${ }^{1}$, and Alyson Wylie ${ }^{2}$, Ben Seipel ${ }^{3}$, \\ and Kevin Buffardi ${ }^{4}$ \\ ${ }^{1}$ Department of Nutrition and Food Science, CSU, Chico \\ ${ }^{2}$ Center for Healthy Communities, CSU, Chico \\ ${ }^{3}$ School of Education, CSU, Chico \\ ${ }^{4}$ Department of Computer Science, CSU, Chico
}

\begin{abstract}
The prevalence of obesity among children in the United States is a major health concern and is associated with increased health risks. The goal of this intervention was to develop and pilot test strategies based on mindful eating practices that foster healthy eating behaviors among elementary school children and their families. Mindful eating practice engages all of ones senses and has been shown to decrease impulsive food decisions and increase awareness of hunger and fullness cues. Using Experiential Learning Theory (ELT), class interventions and take-home activities were developed to teach mindful eating strategies to Hispanic and non-Hispanic $3^{\text {rd }}$ through $5^{\text {th }}$ grade elementary school children and their parents. The results of this research will: 1) contribute to the scientific literature regarding the impact of mindful eating practice on children's eating behavior, 2) provide an alternate strategy for weight loss and management among children, and 3) provide parents and teachers with the knowledge and skills necessary to promote mindful eating among children and families.

(C) 2016 Californian Journal of Health Promotion. All rights reserved.

Keywords: childhood obesity, mindful eating, Experiential Learning Theory, eating behaviors, elementary school children
\end{abstract}

\section{Introduction}

Since 1980, the prevalence of obesity among young children has doubled, and the current rates of childhood overweight and obesity remain a troubling trend and major public health concern (Pelone, et al., 2012). According to the 2011-2012 NHANES data, 31.8\% of children ages 2-19 are either overweight or obese and $16.9 \%$ are obese (Ogden, Carroll, Kit, \& Flegal, 2014). The 2010 summary of health indicators for California from the Pediatric Nutrition Surveillance survey (PedNSS) shows that 23.3\% of children ages 5 to $<20$ are above the $95^{\text {th }}$ percentile (Years, 2010). Children and adolescents who are overweight or obese have been associated with an increased risk of negative short-term health problems and tracking of obesity into adulthood (Ogden, 2012) including the risk of premature mortality or lasting health conditions from developing type 2 diabetes mellitus, asthma, certain cancers, depression, stroke, coronary heart disease and hypertension (Pelone, et al., 2012; Reilly \& Kelly, 2011; Babey, Hastert, Wolstein, \& Diamant, 2010).

Obesity is more common among children and adolescents from families of lower socioeconomic status. Between the years of 2001 to 2007, the disparity in the prevalence of obesity between families with incomes at or above $300 \%$ of the federal poverty line and families with incomes below the line increased from a difference of 7 percentage points to a difference of 15 percentage points. Further, the prevalence of overweight and obesity is disproportionately distributed amongst ethnic groups, and in California the rate of increase has been more noticeable among Latinos and 
African Americans (Babey, Hastert, Wolstein, \& Diamant, 2010).

It is critical to develop and implement early intervention programs to teach skills aimed at promoting a healthy relationship with food and prevention of weight gain. Mindful eating practice is one strategy that may help develop healthful eating behaviors and manage weight. Before examining mindful eating practice, it is important to review ELT which was the theoretical framework for developing the mindful eating intervention program.

\section{Experiential Learning Theory}

David Kolb's Experiential Learning Theory (ELT) works on two levels: a four stage cycle of learning (Figure 1) and four separate learning styles. The four learning styles include 1) diverging (feeling and watching), 2) assimilating (watching and thinking), 3) converging (doing and thinking), and 4) accommodating (doing and feeling) (Kolb, 1984). ELT posits that experience is transformed into knowledge through concrete experiences and abstract conceptualization. Exposure to concrete experiences encourages students to reflect on their observations and then assimilate their reflections into abstract concepts. The newly assimilated concepts then influence future experiences. Experiential learning allows students with different primary learning styles to more easily assimilate new concepts in a way that makes sense to them. According to Kolb "Learning is the process whereby knowledge is created through the transformation of experience" (Kolb, 1984). For this reason, all intervention activities were hands-on and practice-based.

\section{Development of Mindful Eating Lessons and Materials}

Mindful eating encourages the practice of paying purposeful attention to body-related sensations as well as thoughts and feelings about food. This attitude of awareness and acceptance can strengthen the ability to notice internal regulatory cues and decrease the tendency toward avoiding negative emotions through distractions such as emotional eating (Alberts, Thewissen \& Raes, 2012). Though most mindful

\section{Figure 1}

\section{Kolb's Experiential Learning Cycle}

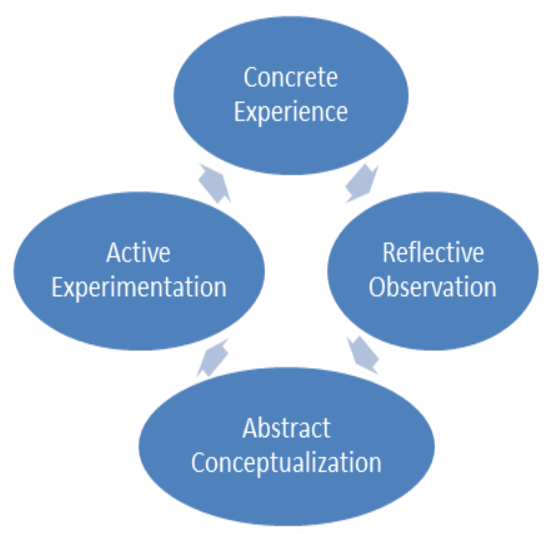

eating studies to date have focused on the treatment of disordered eating behaviors and weight loss, the positive results indicate that training children and adolescents to practice mindful eating skills that promote focused awareness of the whole eating experience could prove to be an effective foundation for interventions aimed at the prevention and treatment of childhood obesity.

"Foodie U: the impact of a pilot mindful eating intervention on behaviors among children and families" is a seed grant project of the Agricultural and Food Research Initiative (AFRI) program funded through the United States Department of Agriculture (USDA). The primary goal of the intervention was to develop and pilot-test strategies based on mindful eating practice for fostering healthy food-related behaviors and the prevention of weight gain. The intervention targeted Hispanic and nonHispanic, low-income, $3^{\text {rd }}-5^{\text {th }}$ grade students and their families in a school in northern California. Activities and lessons were implemented through the Center for Healthy Communities (CHC) in order to coordinate and facilitate scheduling of parent focus groups, classroom activities, and parent workshops. Lesson topics centered around mindful eating practices of enjoying sensory aspects of food, hunger and fullness awareness, emotional awareness, preparing and eating meals together as a family, limiting environmental distractions that promote 
mindless eating, and participating in a cultural feast. When possible, food tastings and takehome activity kits emphasized cultural food items and fresh local produce highlighted by the Harvest of the Month (HOTM) program.

The sequence and type of activities and exercises developed for each of the six lessons was based in part on the principles and components of the Mindfulness-Based Eating Awareness Training (MB-EAT) curriculum. MB-EAT was originally designed for use among adults with problematic eating behaviors such as binge eating disorder, necessitating that the curriculum be adapted for a more general application and a younger audience. In the absence of an available complete mindful eating curriculum for this age group, the specific inclass activities for this intervention were inspired by a variety of existing resources and were developed under the guidance of a mindful eating professional. Latino bilingual/bicultural CHC program managers and staff assisted in translating take-home materials into Spanish.

Parent and guardian buy-in was critical to this study design, which emphasizes in-home practice with family. Therefore, parent and guardian input was valuable in determining whether proposed activities are culturally appropriate and acceptable, and to obtain a realistic gauge of how often mindful shopping, cooking, and eating activities can be practiced within the home during the school year among our specific target population. For this reason, parent input from focus groups were considered during the development of classroom activities, take-home family activity kits, and parent workshops.

\section{Mindful Eating Lessons and Activities in Classroom}

Classroom sessions were 45-60 minutes long and took place once a month for a total of six months at the intervention school. Each month, trained university students implemented a different mindful eating activity aligned with that month's theme and distribute relevant takehome activity kits. Teachers remained in the classroom to facilitate behavior management and were encouraged to participate in activities including the food tastings (Table 1).

\section{Mindful Eating Practice among Parents and Children at Home}

Take-home activity kits were provided after each in-class lesson to promote the practice of mindful eating activities in the home with parents and guardians. These kits included an activity or discussion card, a recipe, and/or nonperishable items. Language appropriate activity kits were prepared prior to the day the corresponding lesson was implemented and distributed to students by the end of the day the lesson was taught. Students were encouraged to practice the same mindful eating exercise at home with their parents by using the activity kits. Incentives such as recipe books, pencils, or magnets were provided if the parent survey was returned, the original take-home activity was practiced and at least one challenge activity was completed.

\section{Focus Group Interviews}

Pre-intervention focus group interviews explored cultural food-related traditions, current perceptions of mindful eating, and parental input regarding the feasibility and appropriateness of suggested take-home activities for the intervention (Table 2). Post-intervention parent focus groups were also held to assess how well the intervention was received and to increase understanding of socio-cultural factors that may affect the future implementation of the intervention with similar populations.

\section{Reflections}

Implementing a project of this scale required much support from the school staff. In addition to creating a schedule for our lessons that fit with the regular school schedule, the school staff also worked with us to schedule us into typically well-attended school-wide events (back-toschool meetings, parent-teacher conference days, open-house nights, etc.) so that we could connect with as many parents/guardians as possible. They coordinated with researchers to ensure that accurate demographic information was obtained for all students, they sent additional flyers home to parents, sent email and phone call reminders home to parents. The 
Pierson, S., Goto, K., Giampaoli, J., Wylie, A., Seipel,B., Buffardi, K. /Californian Journal of Health Promotion 2016, Volume 14, Issue 3, 70-76.

school secretaries also helped by calling individual parents who had completed the surveys in order to distribute gift card incentives

\section{Table 1.}

Monthly Class Lessons and Take Home Activities

Lesson Topics and Classroom Activities

Lesson 1: Master Mindless Munching

Students explore how their daily environment can

influence eating choices and behaviors. Activities focus on awareness of factors that influence portion size.

\section{Lesson 2: Sensational Senses}

Students practice noticing sensory characteristics of dried herbs/spices and are encouraged to make connections to associated memories. Students follow a guided worksheet to explore all sensory attributes of a food and discus the value of taking time to enjoy all that a food has to offer.

Lesson 3: Getting to Know Hunger and Fullness Students discuss awareness of the feeling and meaning of hunger and fullness cues. Students practice using a visual hunger and fullness scale to determine an appropriate snack portion and to notice changes in their hunger/fullness cues as they eat.

\section{Lesson 4: Emotions Inside Out}

Students participate in a brief group discussion to address the differences between physical and emotional needs and the potential influence of emotions of eating behaviors. Students practice mindfulness-based visualization exercises to increase non-judgmental awareness of emotions.

\section{Lesson 5: Planting Seeds of Mindfulness}

Students will identify the resources and individuals involved in producing one food item. Students will practice a guided mindful eating exercise aimed to increase awareness and appreciation of the resources people who produced the food item. Students will plant a seed and identify the resources it will need to grow.

\section{Lesson 6: Cultural Celebration Feast}

Students prepare culturally inspired healthy foods.

Students create a pleasant dining area. Students and parents practice mindful eating skills introduces in previous lessons such as evaluating hunger and appreciating sensory characteristics of the tastings and
Take Home Activities

Portion Distortion Activity

Students and family members serve the amount of

popcorn they would each normally eat into both

large and small bowls, then compare the measured amount of food in each dish.

Five Senses Snack Activity

Students and family members utilize the same worksheet used in class to explore the sensory attributes of a food. Students are encouraged to lead their parents through tastings and discuss with their family the value of food enjoyment.

\section{Hunger and Fullness Scale}

Students teach their family members how to use the hunger and fullness scale to evaluate hunger level prior to eating a meal, select an appropriate initial portion, and use the scale to monitor changes in hunger/fullness cues during and after the meal.

\section{Mindful Choices Interview}

Students or family members select three different food options and each family member selects one that they would most like to eat. Each person takes turns interviewing another person about what thoughts and emotions led them to make that choice.

\section{Nurturing Seeds of Mindfulness}

Students will receive as seed packet with care and transplanting instructions. The family member and student will visit a farmer's market, grocery store or nd library together to answer questions about the aresources and individuals involved in the "life story" of the plant they are growing.

\section{Family Cultural Celebration Meal}

Students and family members will select and work together to prepare a culturally relevant meal.

Students will help decorate set up the dining area. An informal discussion guide will be provided to encourage the sharing of cultural and family 
the resources involved in bringing them to the table. traditions.

Table 2.

Pre-Intervention Parent Focus Group Questions and Probes

Questions Probes

What social-cultural influences play a role in the participant's childhood?

Do the cultural food habits parents have growing up play a role currently in their child feeding practices?

What are the perceptions of mindless eating among participants?

Does mindful eating play a role in their culture and How does it reflect in their family eating patterns?
- What mealtime traditions have you tried to keep going?

- Is it beneficial to keep your food culture alive?

- What does mealtime in your family look like?

- What are some of your current eating practices that may influence your "mindless" eating?

- Are there any environmental influences that may cause you to eat mindlessly?

- Has anyone heard about mindful eating? What does it mean to you?

- Based on the definition, is it something that you practice at home?

- How do you feel when preparing food?

- How do you think your child may benefit from mindful eating? to those parents who did not attend during the times our staff was available on site. Since gift cards require an adult signature in order to distribute them, they worked great as an incentive to get the parents attention. Prior to beginning the intervention, we met frequently with the principal and once with all of the teachers to introduce our project. Prior to each lesson, the lead graduate researcher communicated with teachers via email regarding the lesson topic and activities, foods that would be tasted, and a reminder to collect surveys and activity sheets from students.

This research will contribute knowledge to the scientific literature regarding the impact of mindful eating practice on children's eating behaviors. Currently, there is a dearth of literature on this important subject. Secondly, mindful eating practice can offer an alternative

strategy for weight loss and management in children as opposed to the traditional dietcentered strategies which have not proven effective in the long-term. Teaching children positive eating behaviors during childhood can set them up with healthy eating habits for life. Children can have better long-term health outcomes if they develop a positive relationship to food, along with a balanced approach to eating. Third, mindful intervention lessons and activities provide parents and teachers with the knowledge, skills, and resources for promoting and encouraging mindful eating among children and families. Finally, this research can reveal 
Pierson, S., Goto, K., Giampaoli, J., Wylie, A., Seipel,B., Buffardi, K. /Californian Journal of Health Promotion 2016, Volume 14, Issue 3, 70-76.

differences in mindful eating attitudes and practice between Hispanic and non-Hispanic families. This knowledge can allow future researchers to focus on key cultural beliefs that may enhance or detract from mindful eating practice.

\section{References}

Alberts H.J.E.M., Thewissen, R., \& Raes, L. (2012). Dealing with problematic eating behaviour. The effects of a mindfulness-based intervention on eating behaviour, food cravings, dichotomous thinking and body image concern. Appetite, 58(3):847-851.

Babey, S.H., Hastert, T a., Wolstein, J., \& Diamant, A.L. (2010). Income disparities in obesity trends among California adolescents. American Journal of Public Health, 100(11):2149-2155.

Kolb, D. A. (1984). Experiential learning: Experience as the source of learning and development (Vol. 1). Englewood Cliffs, NJ: Prentice-Hall.

Ogden, C.L. Prevalence of obesity and trends in body mass index among US children and adolescents, 1999-2010. (2012). Journal of the American Medical Associaytion, 307(5):483.

Ogden, C.L., Carroll, M.D., Kit, B.K., \& Flegal, K.M. (2014). Prevalence of childhood and adult obesity in the United States, 2011-2012. Journal of the Ameerican Medical Association, 311(8):806-814.

Pelone, F., Specchia, L., Veneziano, M.A., Capizzi, S., Bucci, S., Mancuso, A., \& Ricciardi, A. (2012). Economic impact of childhood obesity on health systems: A systematic review. Obesity Review, 13(5):431-440.

Years, C.A. (2010). Pediatric Nutrition Surveillance California Run Date: Summary of Health Indicators 2010 Pediatric Nutrition Surveillance California Run Date : Summary of Health Indicators. 
Pierson, S., Goto, K., Giampaoli, J., Wylie, A., Seipel,B., Buffardi, K. /Californian Journal of Health Promotion 2016, Volume 14, Issue 3, 70-76.

\author{
Author Information \\ Shannon Pierson \\ Department of Nutrition and Food Science, CSU, Chico \\ $400 \mathrm{~W} .1^{\text {st }}$ St., Chico, CA, 95929-0002 \\ spierson2@mail.csuchico.edu \\ (760) 514-7100 \\ Keiko Goto, $\mathrm{PhD}$ \\ Department of Nutrition and Food Science, CSU, Chico \\ 400 W. $1^{\text {st }}$ St., Chico, CA, 95929-0002 \\ kgoto@csuchico.edu \\ (530) 898-6767 \\ * Joan Giampaoli, PhD, RDN \\ Department of Nutrition and Food Science, CSU, Chico \\ $400 \mathrm{~W} .1^{\text {st }}$ St., Chico, CA, 95929-0002 \\ jgiampaoli@csuchico.edu \\ (530) 898-6410
}

\author{
Alyson Wylie \\ Center for Healthy Communities, CSU, Chico \\ 25Main St., Suite 101, Chico, CA 95929-0235 \\ awylie@csuchico.edu \\ (530) 898-3311
}

Ben Seipel, PhD

School of Education, CSU, Chico

$400 \mathrm{~W} .1^{\text {st }}$ St., Chico, CA, 95929-0222

bseipel@csuchico.edu

(530) 898-6421

Kevin Buffardi, $\mathrm{PhD}$

Department of Computer Science, CSU, Chico

$400 \mathrm{~W} .1^{\text {st }}$ St., Chico, CA, 95929-0410

kbuffardi@csuchico.edu

(530) 898-5617

* Corresponding Author 